Service social

\title{
La prostitution, une affaire de sens, par Jean-Guy Nadeau, Montréal, Fides, 1987, 469 pages.
}

\section{Germain Trottier}

Volume 36, numéro 2-3, 1987

Approches intégrées

URI : https://id.erudit.org/iderudit/706378ar

DOI : https://doi.org/10.7202/706378ar

Aller au sommaire du numéro

Éditeur(s)

École de service social de l'Université Laval

ISSN

1708-1734 (numérique)

Découvrir la revue

Citer ce compte rendu

Trottier, G. (1987). Compte rendu de [La prostitution, une affaire de sens, par Jean-Guy Nadeau, Montréal, Fides, 1987, 469 pages.] Service social, 36(2-3),

499-501. https://doi.org/10.7202/706378ar d'utilisation que vous pouvez consulter en ligne.

https://apropos.erudit.org/fr/usagers/politique-dutilisation/ 
C'est dans cette partie que l'on voit le plus l'influence du collaborateur Lamoureux (coauteur de L'Intervention communautaire, Montréal, Saint-Martin, 1984) qui décrit les étapes d'une action communautaire : la base du regroupement, l'identification des objectifs et le choix des cibles, la stratégie et les tactiques, les liens avec la population, la négociation et l'art du repli, l'utilisation des médias. On note particulièrement l'importance des médias dans une région semi-rurale.

"Le Comité informa systématiquement les médias des appuis qu'il recevait... Nous avons maintenu un intérêt permanent des médias pour l'ensemble de notre démarche. Dans un milieu comme le nôtre, où les médias régionaux et locaux sont fort importants, cet appui était indispensable. » (p. 76)

On décrit aussi longuement le mode de fonctionnement démocratique du Comité, le rôle des personnes ressources et les niveaux de participation de toutes et chacune. On a même tenu à intégrer des hommes à l'action.

"Pour réaliser cette jonction, nous avons choisi de ne rien faire de spécial pour atteindre les hommes, sinon de ne pas nous engager dans des tactiques de dénonciation globale, style "tous les hommes sont des pornocrates".» (p. 85)

On décrit, après ce long bilan de la lutte, la recherche menée sur les liens entre la violence et la pornographie, financée par le Secrétariat d'État.

Enfin, on termine le cheminement du Centre des femmes en parlant du projet de Centre de préparation à l'emploi mis sur pied au printemps 1986 et de l'intervention en cours sur le transport public en région.

Ainsi, cette œuvre collective se veut un témoignage de femmes qui ont découvert l'action communautaire et politique par l'implication dans le développement d'une région. C'est un message d'espoir.

La lecture est facile et les témoignages de femmes sont nombreux, mais on subit parfois un ton un peu trop didactique voire moralisateur sur l'éthique de l'intervention, les valeurs défendues, l'importance de la démocratie et de la solidarité. C'est sans doute le côté un peu "années 70 " du collaborateur qui ressort à travers une démarche indiscutablement importante et à multiplier dans d'autres régions.

École de service social,

Jean PANET-RAYMOND

Université de Montréal.

La prostitution, une affaire de sens, par Jean-Guy NADEAU, Montréal, Fides, 1987, 469 pages.

Dans la préface de cet ouvrage, Jacques Grand'Maison dit qu' «il y a des livres qui sont plus que des livres tellement ils sont chargés d'humanité, de vérité sur nous-mêmes". L'énoncé paraît taillé sur mesure tant il s'applique bien au livre de Jean-Guy Nadeau. 
Disons au premier abord que ce volume présente le rare avantage de pouvoir être lu dans un ordre qui intéresse le plus le lecteur, chaque partie du volume étant indépendante l'une de l'autre. Dans un style littéraire clair, abondamment descriptif et sous la forme bien adaptée d'une thèse de doctorat en théologie, l'auteur aborde plus spécifiquement le sujet de la prostitution de femmes, à Montréal.

Cinq grandes parties structurent l'étude; la première, de nature plus narrative, rapporte les propos recueillis lors de rencontres avec des femmes " de la gaffe». La deuxième répertorie et analyse les discours féministes et des sciences humaines sur la thématique. La troisième porte sur le rapport que la Bible et la tradition chrétienne entretiennent avec la prostitution. La quatrième partie, composée de cinq chapitres, cerne la structure et la dynamique de prostitution féminine et la dernière partie analyse les enjeux d'ordre éthique, social et pastoral. L'approche méthodologique utilisée est de type praxéologique, c'est-à-dire centrée d'abord sur l'observation systématique d'une pratique pour aboutir à l'émergence d'un rationnel. Même si le courant scientifique nordaméricain ne prise pas plus qu'il n'en faut pareille démarche d'étude, il n'en demeure pas moins qu'elle s'avère efficace dans les domaines réputés hostiles à la recherche, dont la prostitution.

Comme le précise l'auteur avec beaucoup de justesse, la conjoncture fait peut-être en sorte que la prostitution des garçons attire actuellement plus l'attention que la prostitution féminine mais cette dernière reste la plus répandue dans nos sociétés et la base classique du discours connu sur la prostitution.

Deux portraits types de la femme prostituée se profilent dans l'ouvrage : la fille des bars, des clubs, du trottoir, plus visible et souvent plus démunie que la "call-girl " qui elle, se fait plus discrète, plus prudente et qui choisit de filtrer sa clientèle par l'intermédiaire d'annonces de journaux et/ou d'agences spécialisées en la matière. L'une et l'autre, sous une même étiquette de "putain ", vendent du sexe au prix de leur identité et de leur humanité. "Être prostituée, ce n'est pas tellement un métier de sexe, c'est plutôt une façon d'être regardée différemment, d'être rejetée et même de se sentir différente. " (A., dans Claude Jaget, Une vie de putain)

L'abus, l'exploitation par tous genres d'hommes marquent la destinée de ces femmes. Le passé familial lourd, le rejet, la méfiance, la solitude, l'enfermement, les tendances suicidaires, la quête d'amour, le désespoir les habitent en commun et les stigmatisent. La finalité de l'agir loge quelque part entre l'argent et la quête de tendresse humaine.

Par ses procédés de réflexion et d'analyse, l'auteur propose une définition intéressante de la rencontre prostitutionnelle :

"En majeure, il s'agit d'un rapport objectivant, socialement organisé, contrôlé, exploité et stigmatisant pour la femme prostituée, où des personnes échangent, sous un mode anonyme, souvent violent, irresponsable, imaginaire, et sans risque pour le client, une double quête de disponibilité. D'une part une relation sexuelle et une jouissance souvent simulée par la prostituée, gage de la virilité du client; d'autre part une somme d'argent qui est aussi gage de valeur personnelle et sociale pour la prostituée. 
En mineure, et les éléments précédents y demeurant présents, ce rapport se révèle parfois l'occasion d'une rencontre réelle entre deux personnes qui sont aussi en quête d'une présence humaine".

Ici, la prostitution prend un sens tactique à la fois sexuel, économique et social qui décape la conscience individuelle et sociale et nous projette en dehors des clichés habituels, au-delà de l'interprétation réduite de la " passe pour l'argent ". Prostituée - client - souteneur ne sont plus les seuls en cause, mais un large milieu socio-économique et culturel soutient, organise, promeut et contrôle leur relation.

L'œuvre de Nadeau permet l'ouverture d'une parenthèse plus élargie sur un segment de la condition humaine agissante.

École de service social,

Germain TrottieR

Université Laval.

The One-Parent Family in the 1980s. Perspectives and Annotated Bibliography 1978-1984, par Benjamin SCHLESINGER, Toronto, University of Toronto Press, 1985, 284 pages.

L'ouvrage de Schlesinger est d'un genre littéraire relativement rare en Amérique du Nord et quasi-inexistant en Europe. Il n'en est que plus précieux, et ce tout particulièrement pour les chercheurs ou étudiants européens qui méconnaissent le plus souvent l'énorme somme de travaux produits par leurs collègues d'outre-Atlantique. En effet, il s'agit d'un inventaire aussi large et exhaustif que possible des publications (ouvrages, travaux de recherche et articles) concernant les familles monoparentales au Canada et aux États-Unis. On doit avant tout saluer cette initiative de l'auteur, qui depuis la fin des années soixante tient à jour et réédite régulièrement cette bibliographie annotée. On ne peut espérer un outil plus idéal pour traverser cette énorme production nord-américaine. Ainsi conçu, ce document est un outil de travail tout à fait irremplaçable pour toute personne, étudiant, chercheur ou enseignant, intéressé par les questions concernant l'évolution des structures familiales.

La première édition de cet ouvrage date de 1969. Trois autres l'ont suivie et comptaient 750 références bibliographiques annotées portant sur le thème et couvrant la période 1930-1978. La présente édition complète les précédentes en proposant, d'une part, une bibliographie commentée de 490 nouvelles références publiées entre janvier 1978 et juin 1984, et d'autre part, des synthèses de littérature concernant des aspects particuliers liés à la monoparentalité. Ces synthèses rédigées par L. Davids, A.M. Ambert, S.M.H. Hanson, G. Nelson et l'auteur lui-même enrichissent considérablement ce document et montrent l'étendue des questions associées à ce phénomène. Les auteurs ont su dépasser la catégorie elle-même pour aborder des sujets voisins, comme la recomposition des familles, le remariage, ou encore le sujet trop souvent négligé des parents 